Article

\title{
Paradox-Proof Utility Functions for Heavy-Tailed Payoffs: Two Instructive Two-Envelope Problems
}

\section{Michael R. Powers}

Department of Finance, School of Economics and Management, and Schwarzman Scholars Program, Tsinghua University, Beijing 100084, China; E-Mail: powers@ sem.tsinghua.edu.cn;

Tel.: +86-10-6279-5565.

Academic Editor: José Garrido

Received: 5 October 2014 / Accepted: 22 December 2014 / Published: 19 January 2015

\begin{abstract}
We identify restrictions on a decision maker's utility function that are both necessary and sufficient to preserve dominance reasoning in each of two versions of the Two-Envelope Paradox (TEP). For the classical TEP, the utility function must satisfy a certain recurrence inequality. For the St. Petersburg TEP, the utility function must be bounded above asymptotically by a power function, which can be tightened to a constant. By determining the weakest conditions for dominance reasoning to hold, the article settles an open question in the research literature. Remarkably, neither constant-bounded utility nor finite expected utility is necessary for resolving the classical TEP; instead, finite expected utility is both necessary and sufficient for resolving the St. Petersburg TEP.
\end{abstract}

Keywords: two-envelope paradox; dominance reasoning; von Neumann-Morgenstern utility; heavy-tailed payoffs; boundedness

\section{Introduction}

We identify restrictions on a decision maker's von Neumann-Morgenstern (VNM) utility function that are both necessary and sufficient to preserve dominance reasoning (i.e., $A|C \succ B| C \forall C \Rightarrow A \succ$ $B$ ) in each of two versions of the Two-Envelope Paradox (TEP). For the classical TEP of [1] and [2] with continuous payoffs, the utility function must satisfy a certain recurrence inequality for at least one continuous interval. For the St. Petersburg TEP of [3], also with continuous payoffs, the utility function must be asymptotically bounded above by a power function, and this can be tightened to a constant by arguments similar to those of [4]. For discrete payoffs, analogous results apply. 
Determining the weakest conditions for dominance reasoning to hold in the classical and the St. Petersburg TEPs settles an open question posed by [3]. Remarkably, the analysis shows that neither constant-bounded utility nor finite expected utility is necessary for resolving the classical TEP, whereas finite expected utility is both necessary and sufficient for resolving the St. Petersburg TEP.

\section{The Classical Two-Envelope Paradox}

\subsection{Naive Form}

Numerous early variations of the TEP exist. One formulation is that of [5], in which two men bet on the value of their respective neckties, with both neckties going to the man with the less valuable item. Another formulation, involving explicit distributions of monetary values printed on both sides of randomly drawn playing cards, is found in [6] and attributed to the physicist Erwin Schrödinger. In the simplest form of the classical TEP (see [1]), a risk-neutral decision maker is shown two identical envelopes, one of which contains a check for $X$ units of money, and the other a check for $2 X$ units, where $X$ is an unknown positive real number. The two envelopes then are dropped into a box, from which the decision maker draws one at random. At this point, the decision maker is asked whether he or she would like to keep the chosen envelope, or trade for the other one. Seeing no benefit from trading (because of the obvious symmetry of the alternatives), the decision maker then opens the chosen envelope to find a check for $Y=y$ units. Asked again whether he or she would like to trade envelopes, the decision maker calculates the expected value of the amount in the other envelope, $Z$, as

$$
E[Z \mid Y=y]=(1 / 2)(y / 2)+(1 / 2)(2 y)=1.25 y
$$

and discovers that it is better to trade, because the two possibilities, i.e., $Z=y / 2$ and $Z=2 y$, are presumably equally likely. Paradoxically, the decision maker's behavior is the same for all values of $y$-implying no benefit from opening the envelope-but learning the value of $y$ does in fact change the decision maker's preference. In short, the decision maker's failure to prefer $Z$ to $Y$ a priori is a violation of dominance reasoning.

This naive TEP is typically resolved by observing that $Z=y / 2$ and $Z=2 y$ cannot be equally likely for all positive real numbers $y$, because that would require $Z$ to possess a (nonexistent) uniform probability distribution over the positive real numbers.

\subsection{Enhanced Form}

Nalebuff offered a more sophisticated variation of the classical TEP in which the random gain $X$ is drawn from a distribution function (known to the decision maker) that always implies a positive expected benefit from trading once $Y=y$ has been observed [1], so that

$$
E[Z \mid Y=y]>y
$$


for all possible values of $y$. (Actually, Nalebuff inadvertently claimed only that the expected benefit is nonnegative. His example employs the distribution function in Equation (2) of the present article, with $m=1$ and $p=1-(1 / \sqrt{2})$.) A necessary condition for these results is that

$$
E[Y]=E[Z]=\infty
$$

Such "paradoxical" distribution functions for $X$ can be either continuous or discrete, and [2] offered one example of each:

$$
X \sim f_{X}(x)=\frac{1}{(x+1)^{2}}, x>0
$$

in the continuous case, and

$$
X \sim f_{X}(x)=\frac{1}{3}\left(\frac{2}{3}\right)^{\log _{2}(x)}, x=1,2,4,8, \ldots,
$$

in the discrete case. In the present work, it will be useful to embed each of the above distributions into a natural, two-parameter generalization. For continuous $X$, this is the Pareto II family:

$$
X \sim f_{X}(x)=\frac{\alpha}{\theta}\left(\frac{\theta}{x+\theta}\right)^{\alpha+1}, x>0
$$

for any $\alpha$ in the interval $(0,1]$ and positive $\theta$; whereas for discrete $X$, it is the log negative binomial family:

$$
X \sim f_{X}(x)=\left(\begin{array}{c}
m+\log _{2}(x)-1 \\
m-1
\end{array}\right) p^{m}(1-p)^{\log _{2}(x)}, x=1,2,4,8, \ldots
$$

for any positive integer $m$ and $p$ in the interval $(0,1 / 2]$. Note that although not immediately apparent, the distributions in Equations (1) and (2) are close analogs because the Pareto II distribution with $\theta=1$ is obtained by exponentiating and shifting an exponential random variable, and the log negative binomial distribution with $m=1$ is obtained by exponentiating a geometric random variable (i.e., the discrete analog of the exponential random variable). The characterization of all possible "paradoxical" distributions is beyond the scope of the present work.

Our analysis will focus on the continuous case because it retains the infinitely divisible nature of $X$ from the naive TEP. However, we also will briefly summarize the corresponding results for the discrete case.

\subsection{Continuous Payoffs}

Relaxing the assumption that the decision maker is risk neutral, let the decision maker's VNM utility function be some continuous increasing function $u(\cdot)$ defined for all real numbers greater than or equal to the decision maker's initial wealth, $W$. For $X$ with a Pareto II distribution, it is easy to see that the paradox persists for this generalization if and only if both of the following conditions are satisfied:

$$
E[u(W+Y)]=E[u(W+Z)]
$$

and

$$
E[u(W+Z) \mid Y=y]>u(W+y)
$$


for all positive $y$. In other words, the random variables $Y$ and $Z$ are equally desirable unconditionally (i.e., prior to opening the decision maker's envelope), but $Z$ is strictly preferable to $Y$ conditionally (i.e., after opening the envelope to reveal $Y=y$ ), thereby violating dominance reasoning.

Given that the random variables $Y$ and $Z$ are unconditionally independent and identically distributed, Equation (3) is always true. Therefore, to avoid the inconsistency of Equations (3) and (4) (and thus preserve dominance reasoning), we must require that

$$
E[u(W+Z) \mid Y=y] \leq u(W+y)
$$

or equivalently,

$$
\begin{gathered}
u(W+y / 2) \operatorname{Pr}\{Z=y / 2 \mid Y=y\}+u(W+2 y) \operatorname{Pr}\{Z=2 y \mid Y=y\} \\
\leq u(W+y)
\end{gathered}
$$

for at least one continuous interval of $y$. If Equation (5) does not hold for such an interval, then it constitutes an event "of probability zero" that has no practical impact on the decision maker's behavior. Proposition 1 provides necessary and sufficient restrictions on the utility function to satisfy this inequality, and is proved in the Appendix (as are all subsequent propositions).

Proposition 1: For any continuous increasing utility function and distribution parameters $\alpha$ and $\theta$, dominance reasoning is preserved if and only if the recurrence inequality

$$
\begin{gathered}
2^{\alpha}(y+\theta)^{\alpha+1} u(W+y / 2)+(y+2 \theta)^{\alpha+1} u(W+2 y) \\
\leq\left[(y+2 \theta)^{\alpha+1}+2^{\alpha}(y+\theta)^{\alpha+1}\right] u(W+y)
\end{gathered}
$$

holds for at least one interval of positive $y$.

From the expression for $\operatorname{Pr}\{Z=y / 2 \mid Y=y\}$ employed in the proof of Proposition 1, it is easy to show that this probability is increasing over $y$ for fixed $\alpha$ and $\theta$, from a lower bound of $1 / 3$ at $y=0$ to an upper bound of $2^{\alpha} /\left(2^{\alpha}+1\right)$ as $y \rightarrow \infty$. Consequently, one can use the lower bound to derive the following sufficient (i.e., stronger) condition for Equations (5) and (6):

$$
u(W+y / 2)+2 u(W+2 y) \leq 3 u(W+y),
$$

and the upper bound to derive the following necessary (i.e., weaker) condition:

$$
2^{\alpha} u(W+y / 2)+u(W+2 y) \leq\left(2^{\alpha}+1\right) u(W+y) .
$$

These last two conditions are more readily interpreted than Equation (6) because their corresponding equalities describe concave-downward curves of the forms

$$
u(W+y)=-a y^{-1}+b
$$

and

$$
u(W+y)=a y^{\alpha}+b,
$$

respectively, where $a>0$ and $b$ are real constants determined by any two points satisfying the indicated equation. Note that these results follow from straightforward applications of recurrence-relation 
techniques, and are easily confirmed by substituting the right hand sides of Equations (9) and (10) into the equalities associated with Equations (7) and (8), respectively. Thus, if the piecewise-linear functions passing through $u(W+y / 2), u(W+y)$ and $u(W+2 y)$ are bounded below locally by the hyperbolas of Equation (9) for all $y$ in the indicated interval, then Equation (6) must hold. Here, by "bounded below locally," we mean that if $a$ and $b$ are chosen such that $u(W+y / 2)=-a(y / 2)^{-1}+b$ and $u(W+2 y)=-a(2 y)^{-1}+b$, then $u(W+y) \geq-a y^{-1}+b$. Furthermore, if Equation (6) holds, then the piecewise-linear functions passing through $u(W+y / 2), u(W+y)$ and $u(W+2 y)$ must be bounded below locally (like stated above) by the translated power functions of Equation (10) for all $y$ in the indicated interval.

Although the function in Equation (9) is bounded above by the constant $b$, it is important to note that Proposition 1 does not require that the decision maker's utility function be bounded by a constant, or even that the expected utility be finite. For example, the unbounded piecewise utility function

$$
u(W+y)=\left\{\begin{array}{c}
4 y-4,0 \leq y \leq 1 / 2 \\
-y^{-1}, 1 / 2<y \leq 3 \\
(1 / 9) y-2 / 3, y>3
\end{array}\right.
$$

generates, for $W=0$, infinite expected utility because it is linear for values greater than 3, but satisfies Equation (7) on the interval $(1,3 / 2$ ] because it possesses the form Equation (9) for values greater than $1 / 2$ and less than or equal to 3 . The various constants in the function are chosen to confer a high degree of smoothness (i.e., continuous differentiability).

\subsection{Discrete Payoffs}

Letting $X$ have a log negative binomial distribution yields the following analog of Proposition 1 .

Proposition 2: For any continuous increasing utility function and distribution parameters $m$ and $p$, dominance reasoning is preserved if and only if the recurrence inequality

$$
\begin{gathered}
u(W+y / 2)+\frac{\log _{2}(y)+m-1}{\log _{2}(y)}(1-p) u(W+2 y) \\
\leq\left[\frac{\log _{2}(y)+m-1}{\log _{2}(y)}(1-p)+1\right] u(W+y)
\end{gathered}
$$

holds for at least one $y=2,4,8,16, \ldots$.

\section{The St. Petersburg Two-Envelope Paradox}

\subsection{Conditional Independence}

Chalmers introduced the St. Petersburg TEP to isolate the paradoxical characteristics of the classical TEP from any conditional statistical dependence between the payoff amounts [3]. In this new problem, a risk-neutral decision maker is shown two identical envelopes: one containing a check for $Y$ units of 
money, and the other a check for $Z$ units, where $Y$ and $Z$ are independent and identically distributed random variables with infinite means. A priori, the decision maker is indifferent between the two envelopes because

$$
E[Y]=E[Z]=\infty .
$$

However, upon opening one envelope to find a check for $Y=y$ units, the decision maker would rather trade envelopes because

$$
E[Z \mid Y=y]=E[Z]=\infty>y
$$

As in the classical TEP, dominance reasoning fails.

Although Chalmers worked with random variables similar to that of the original St. Petersburg Paradox (i.e., such that $Y$ and $Z$ possess a shifted $\log$ negative binomial distribution with $m=1$ and $p=1 / 2$ ), the main thrust of his argument can be accomplished with either the Pareto II or log negative binomial distribution. As before, we will focus on the continuous case, and then summarize results for the discrete case.

\subsection{Continuous Payoffs}

Letting the decision maker's VNM utility function be some continuous increasing function and $Y$ and $Z$ be Pareto II random variables, the paradox persists if and only if both of the following conditions are satisfied:

$$
E[u(W+Y)]=E[u(W+Z)]
$$

and

$$
E[u(W+Z) \mid Y=y]=E[u(W+Z)]>u(W+y)
$$

for all positive $y$. Since $Y$ and $Z$ are independent and identically distributed, Equation (11) is always true. Therefore, to avoid the inconsistency of Equations (11) and (12), we must have

$$
E[u(W+Z) \mid Y=y]=E[u(W+Z)] \leq u(W+y)
$$

for at least one finite interval of $y$. This occurs if and only if the expected values on the left hand side of the inequality are finite, in notable contrast to the conditions for resolving the classical TEP. Proposition 3 provides necessary and sufficient restrictions on the utility function for Equation (13) to hold.

Proposition 3: For any continuous increasing utility function and distribution parameters $\alpha$ and $\theta$, dominance reasoning is preserved if and only if

$$
\lim _{y \rightarrow \infty} \frac{u(W+y)}{y^{\alpha}}=0 .
$$

Thus, to satisfy Equation (13), the utility function must be bounded above asymptotically by the indicated power function. Although [3] did not explicitly pursue a line of reasoning similar to that of [4], it is easy to argue that, for increasingly heavy-tailed distributions $Y$ and $Z$, the decision maker's utility function must be bounded above by a constant. Specifically, if one replaces $Y$ and $Z$ with $Y^{\prime}=e^{Y}-1$ and $Z^{\prime}=e^{Z}-1$, then one must replace Equation (14) with

$$
\lim _{y \rightarrow \infty} \frac{u(W+y)}{(\ln (y+1))^{\alpha}}=0 .
$$


Likewise, replacing $Y$ and $Z$ with $Y^{\prime \prime}=e^{Y^{\prime}}-1$ and $Z^{\prime \prime}=e^{Z^{\prime}}-1$ causes Equation (14) to become

$$
\lim _{y \rightarrow \infty} \frac{u(W+y)}{(\ln (\ln (y+1)+1))^{\alpha}}=0
$$

and the pattern may be continued indefinitely.

\subsection{Discrete Payoffs}

Using $Y$ and $Z$ with a log negative binomial distribution gives the following analog of Proposition 3. Proposition 4: For any continuous increasing utility function and distribution parameters $m$ and $p$, dominance reasoning is preserved if and only if

$$
\lim _{y \rightarrow \infty} \frac{u(W+y)}{y^{\log _{2}(1 /(1-p))}}=0 .
$$

This asymptotic upper bound can be tightened to a constant for increasingly heavy-tailed random variables by replacing $Y$ and $Z$ with $Y^{\prime}=2^{Y}$ and $Z^{\prime}=2^{Z}, Y^{\prime \prime}=2^{Y^{\prime}}$ and $Z^{\prime \prime}=2^{Z^{\prime}}$, etc.

\section{Conclusions}

In the present work, we have identified restrictions on a decision maker's VNM utility function that are both necessary and sufficient to resolve each of two TEPs: the classical TEP of [1] and [2], and the St. Petersburg TEP of [3]. These results determine the weakest conditions for preserving dominance reasoning in the two TEPs, thereby settling an open question posed by [3]. Interestingly, neither constant-bounded utility nor finite expected utility is necessary for resolving the classical TEP. This is somewhat remarkable in light of the assertion — common to many discussions of the TEP — that dominance reasoning fails because either utility or expected utility diverges to infinity. In contrast, finite expected utility is both necessary and sufficient for resolving the St. Petersburg TEP.

\section{Acknowledgments}

The author thanks the AXA Research Fund for its generous support.

\section{Appendix}

\section{Proof of Proposition 1:}

From the appendix of [2], we know that, for the continuous case,

$$
\operatorname{Pr}\{Z=y / 2 \mid Y=y\}=\frac{f_{X}(y / 2)}{2 f_{X}(y)+f_{X}(y / 2)}
$$

for all positive $y$, from which it follows that

$$
\operatorname{Pr}\{Z=y / 2 \mid Y=y\}=\frac{\frac{\alpha}{\theta}\left(\frac{\theta}{y / 2+\theta}\right)^{\alpha+1}}{\frac{2 \alpha}{\theta}\left(\frac{\theta}{y+\theta}\right)^{\alpha+1}+\frac{\alpha}{\theta}\left(\frac{\theta}{y / 2+\theta}\right)^{\alpha+1}}
$$




$$
=\frac{2^{\alpha}(y+\theta)^{\alpha+1}}{(y+2 \theta)^{\alpha+1}+2^{\alpha}(y+\theta)^{\alpha+1}} .
$$

Substituting the above expression into Equation (5) and rearranging terms then yields the desired inequality.

\section{Proof of Proposition 2:}

From [2], it is known that, for the discrete case,

$$
\operatorname{Pr}\{Z=y / 2 \mid Y=y\}=\frac{f_{X}(y / 2)}{f_{X}(y)+f_{X}(y / 2)}
$$

for all integers $y$ greater than 1 , which implies

$$
\begin{gathered}
\operatorname{Pr}\{Z=y / 2 \mid Y=y\} \\
\left(\begin{array}{c}
m+\log _{2}(y)-1 \\
m-1
\end{array}\right) p^{m}(1-p)^{\log _{2}(y)}+\left(\begin{array}{c}
m+\log _{2}(y / 2)-1 \\
m-1 \\
m-1
\end{array}\right) p^{m}(1-p)^{\log _{2}(y / 2)} \\
=\frac{1}{\frac{m+\log _{2}(y)-1}{\log _{2}(y)}(1-p)+1} .
\end{gathered}
$$

Substituting this expression into Equation (5) and rearranging terms then yields the desired inequality.

\section{Proof of Proposition 3:}

As noted in the text, Equation (13) is true if and only if the expected values on its left hand side are finite. For fixed values of $\alpha$ and $\theta$, this means

$$
\int_{0}^{\infty} u(W+y) \frac{\alpha}{\theta}\left(\frac{\theta}{y+\theta}\right)^{\alpha+1} d y<\infty
$$

or equivalently,

$$
\lim _{y \rightarrow \infty} \frac{u(W+y)}{y^{\alpha}}=0
$$

\section{Proof of Proposition 4:}

As in the proof of Proposition 3, Equation (13) is true if and only if the expected values on its left hand side are finite. For fixed values of $m$ and $p$, this means

$$
\sum_{y=1,2,4,8, \ldots} u(W+y)\left(\begin{array}{c}
m+\log _{2}(y)-1 \\
m-1
\end{array}\right) p^{m}(1-p)^{\log _{2}(y)}<\infty,
$$

which can be rewritten as

$$
\sum_{k=0}^{\infty} u\left(W+2^{k}\right) \frac{\left(m+\log _{2}\left(2^{k}\right)-1\right) !}{\left(\log _{2}\left(2^{k}\right)\right) !(m-1) !} p^{m}(1-p)^{\log _{2}\left(2^{k}\right)}<\infty .
$$


Canceling factors that do not depend on $k$ and substituting Stirling's approximation for all factorials greater than 0 ! then yields

$$
\begin{aligned}
& u(W+1) \frac{(m-1) !}{0 !}(1-p)^{0}
\end{aligned}
$$

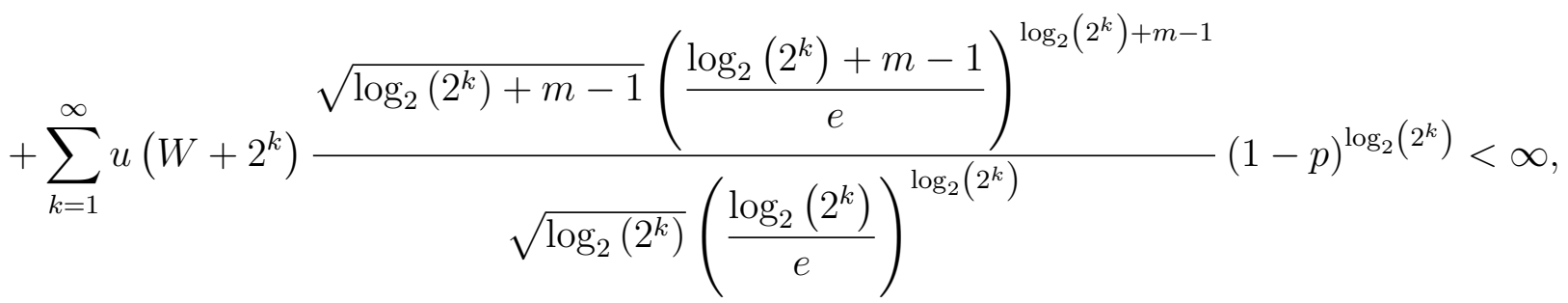

and equivalently,

$$
\sum_{k=1}^{\infty} u\left(W+2^{k}\right)\left(\log _{2}\left(2^{k}\right)\right)^{m-1}(1-p)^{\log _{2}\left(2^{k}\right)}<\infty .
$$

Finally, it follows from the Cauchy condensation test that

$$
\sum_{y=2}^{\infty} u(W+y)\left(\log _{2}(y)\right)^{m-1} e^{-\ln (1 /(1-p)) \log _{2}(y)}<\infty,
$$

which is finite (by the integral test) if and only if

$$
\lim _{y \rightarrow \infty} \frac{u(W+y)}{e^{\ln (1 /(1-p)) \log _{2}(y)}}=0,
$$

or equivalently,

$$
\lim _{y \rightarrow \infty} \frac{u(W+y)}{y^{\log _{2}(1 /(1-p))}}=0
$$

\section{Conflicts of Interest}

The author declares no conflict of interest.

\section{References}

1. Nalebuff, B. The Other Person's Envelope Is Always Greener. J. Econ. Perspect. 1989, 3, 1, 171-181.

2. Broome, J. The Two-Envelope Paradox. Analysis 1995, 55, 1, 6-11.

3. Chalmers, D.J. The St. Petersburg Two-Envelope Paradox. Analysis 2002, 62, 2, 155-157.

4. Menger, K. Das Unsicherheitsmoment in der Wertlehre. J. Econ. 1934, 5, 4, 459-485.

5. Kraitchik, M. Mathematical Recreations; W.W. Norton: New York, NY, USA, 1942.

6. Littlewood, J.E. Littlewood's Miscellany; Bollobás, B., Ed.; Cambridge University Press: Cambridge, UK, 1986.

(C) 2015 by the author; licensee MDPI, Basel, Switzerland. This article is an open access article distributed under the terms and conditions of the Creative Commons Attribution license (http://creativecommons.org/licenses/by/4.0/). 